\title{
基于哈希自注意力端到端网络的三维模型草图检索
}

\author{
赵旭飞1)，潘翔 ${ }^{11^{*}}$ ，刘复昌 ${ }^{2}$ ，张三元 ${ }^{3)}$ \\ 1) (浙江工业大学计算机科学与技术学院 杭州 310023) \\ 2) (杭州师范大学信息科学与工程学院 杭州 311121) \\ 3) (浙江大学计算机科学与技术学院 杭州 310013) \\ (panx@zjut.edu.cn)
}

\begin{abstract}
摘 要: 为了提高草图和三维模型视图嵌人特征的聚类性, 提出一种结合自注意力和哈希正则化约束的特征提取算 法. 首先将三维模型渲染得到二维视图集, 并通过边缘检测在草图和视图之间建立统一的特征描述空间; 然后在共 享权重网络中嵌人自注意力层, 通过结构信息自相关性编码提高草图和视图的聚类性, 避免局部差异性对结果的影 响; 最后对特征进行哈希编码，并嵌人哈希正则化约束和交叉熵损失函数，避免特征值发散. 对基准数据集 SHREC13 和 SHREC14 的实验结果表明, 该算法在哈希自注意力端到端网络的检索准确率方面优于已有的典型算法, 平均准确率性能提高了 $6 \%$.
\end{abstract}

关键词: 三维模型草图检索; 哈希共享权重; 自注意力; 端到端网络

中图法分类号: TP391.41 DOI: 10.3724/SP.J.1089.2021.18548

\section{Hash Self-Attention End-to-End Network for Sketch-Based 3D Shape Retrieval}

\author{
Zhao Xufei $^{1)}$, Pan Xiang ${ }^{1)^{*}}$, Liu Fuchang ${ }^{2)}$, and Zhang Sanyuan ${ }^{3)}$ \\ 1) (College of Computer Science and Technology, Zhejiang University of Technology, Hangzhou 310023) \\ 2) (College of Information Science and Engineering, Hangzhou Normal University, Hangzhou 311121) \\ 3) (College of Computer Science and Technology, Zhejiang University, Hangzhou 310013)
}

\begin{abstract}
To improve the feature clustering of sketches and 3D models, a feature extraction network with self attention and hash regularization is proposed. Firstly, the $3 \mathrm{D}$ model is rendered to obtain the different views. Secondly, the self attention layer is embedded in the shared weight network. In this way, the clustering of sketches and views is improved through the auto-correlation coding of shape structural information. Furthermore, it can avoid the influence of local differences between hand-rendering sketch and view edge. Finally, the algorithm encodes the features with hash regularization constraint and cross entropy loss function to avoid the divergence of eigenvalues. The experimental analysis on SHREC13 and SHREC14 shows that the retrieval accuracy of hash self attention end-to-end network is better than the existing typical algorithms. The mean average precision performance is improved by $6 \%$.
\end{abstract}

Key words: sketch-based 3D shape retrieval; hash shared weights; self-attention; end-to-end network

随着三维扫描设备、三维建模等软硬件技术的 发展，三维模型数量迅猛增长，用户对三维模型的
检索手段却很重视．对于三维模型检索，采用文本 关键字的方法虽然简单, 但是文本描述和三维模

收稿日期: 2020-08-06; 修回日期: 2021-01-10. 基金项目：浙江省自然科学基金(LY19F020031，LY20F020017). 赵旭飞(1994一), 男, 硕士研究生, 主要研究方向为三维模型检索; 潘翔(1977一), 男, 博士, 教授, 博士生导师, CCF 会员, 论文通讯作者, 主要研究 方向为图像处理、三维重建、三维模型检索; 刘复昌(1982-), 男, 博士, 讲师, 硕士生导师, CCF 会员, 主要研究方向为人工智能、 虚拟现实; 张三元(1969-), 男, 博士, 教授, 博士生导师, CCF 会员, 主要研究方向为计算机图形学. 
型内容所存在的语义鸿沟问题使这种检索方法具 有局限性. 基于实例的检索方式需要用户提供已 有模型作为输人，也存在局限性. 与这些检索方法 相比，随着触屏设备和相关技术的成熟，用户可以 通过简单的线条勾勒完成手绘草图的创建，而且 能够有效地表明查询意图. 因此, 三维模型草图检 索引起了研究人员的广泛兴趣 ${ }^{[1-4]}$.

但是，三维模型草图检索是一个具有挑战性 的研究课题，主要原因是草图只由简单线条组成， 而三维模型是由点面组成的高维数据. 另外, 由于 草图绘制具有极强的主观性，同一物体由不同的 人绘制会具有差异性. 因此，如何通过深度学习等 卷积理论建立两者之间的特征映射，提高草图和 三维模型的相似性, 是相关研究需要解决的主要 问题. 对于草图和三维模型的相似性学习问题, 早 期研究主要采用人工设计的特征描述符. Eitz 等 ${ }^{[5]}$ 使用 Gabor 滤波器与特征袋定义获得 Gabor 局部线 性特征的方法，通过局部描述符和草图的量化将 草图表示为视觉特征直方图, 实现草图与视图的 特征映射

近年来, 随着深度学习理论的广泛应用, 研究 人员开始考虑采用卷积网络解决草图和三维模型 的相似性问题. 主要有以下 2 类方法. 一类方法是 分别提取草图和三维模型的深度特征, 然后通过 特征变换网络建立域间的语义映射关系 ${ }^{[6-7]}$. 尽管 在理论上特征变换网络可以通过反向传播实现不 同域之间的特征逼近，但实际上由于 2 个域在特征 空间上的差异性，从而使特征映射得到的检索准 确率并不高. Dai 等 ${ }^{[6]}$ 通过三维模型的尺度不变特 征转换法和卷积网络分别提取三维模型及草图的 原始特征; 然后同时训练 2 个特征变换网络, 实现 跨域特征的分布一致性和域内特征的区分. $\mathrm{Mu}$ 等 ${ }^{[7]}$ 首先将三维模型特征建模为对称正定矩阵, 将草 图特征建模为欧几里得点; 然后将它们同时映射 到高维希尔伯特空间, 实现度量学习. 另一类方法 是通过投影和边缘检测操作, 将三维模型投影渲 染为二维视图集，采用边缘检测将二维视图集提 取为一组边缘线条图, 从而将草图与视图统一到 同一特征描述空间，尽可能地减少跨域差异性和 无用特征的干扰 ${ }^{[8-12]}$. Wang 等 ${ }^{[8]}$ 在此基础上首次提 出采用卷积网络同时学习草图和二维视图集的特 征, 通过损失函数定义域内和域间的相似性, 所取 得的检索准确率优于以往人工选择最佳视图的方 式. Xie 等 ${ }^{[9]}$ 通过 2 个深度卷积网络分别提取草图 和视图集的深度特征; 然后计算二维视图集特征
的瓦瑟斯坦重心表示三维模型, 同时构建度量网 络学习二维视图集的瓦瑟斯坦重心, 实现草图和 三维模型的跨域匹配. Chen 等 ${ }^{[10-11]}$ 首先通过度量 学习网络学习草图与二维视图集的深度特征; 然 后构建跨模态转换网络缩小域间差异性, 增强域 间数据分布之间的整体相关性, 实现最终检索. 他 们在此基础上提出将深度特征转换成哈希编码进 行特征的嵌人; 然后基于视角加权融合二维视图 集特征，提高了整体检索效率和检索准确率. 白静 等 ${ }^{[12]}$ 首先把三维模型渲染为一组二维视图集, 建 立跨域数据的共享数据空间; 然后通过网络权值 共享, 建立端到端的三元度量学习网络, 实现跨域 数据的联合特征映射.

尽管已有研究通过投影和边缘检测等理论把 三维模型转化为和草图类似的表示，可用户绘制 的草图和边缘视图在全局特征上并不具有相似性, 但是草图和边缘视图在形状结构上具有相似性. 因此，本文提出了哈希自注意力端到端网络，用于 提取草图和边缘视图在语义空间约束下的自相关 性特征. 哈希自注意力端到端网络把草图和边缘 视图通过共享权重自注意力层提取形状结构特征, 并在损失函数中嵌人哈希正则化约束特征值，从 而将提取的语义特征转换为哈希编码. 这种表示 方法不仅提高了编码效率, 而且使草图和边缘视 图在特征上更具有聚类性. 本文针对基准数据集 的实验分析表明，哈希自注意力端到端网络可以 得到更高的三维模型草图检索准确率。

\section{1 本文算法}

基于哈希自注意力端到端网络的三维模型草 图检索总体框架如图 1 所示. 首先对三维模型进行 预处理, 将三维模型渲染为多视角的二维视图集, 并通过边缘检测在二维视图和草图之间建立统一 的特征描述空间; 然后在哈希共享权重网络中提 取草图和视图的高层语义特征, 并在共享权重网 络中嵌人自注意力层，提高特征聚类性; 最后将哈 希正则化监督嵌人加权损失函数, 对比度量学习 草图和视图的高层语义特征, 从而得到能够缩小 域间差异性同时，保留共同属性的哈希编码.

\section{1 统一特征描述空间的构建}

三维模型是包含点云等空间位置信息的表征 方式，而二维草图一般只是对被描述物体的简单 线条勾勒的轮廓图, 两者无法直接在同一特征描 述空间进行匹配, 因此需要先对三维模型进行预 


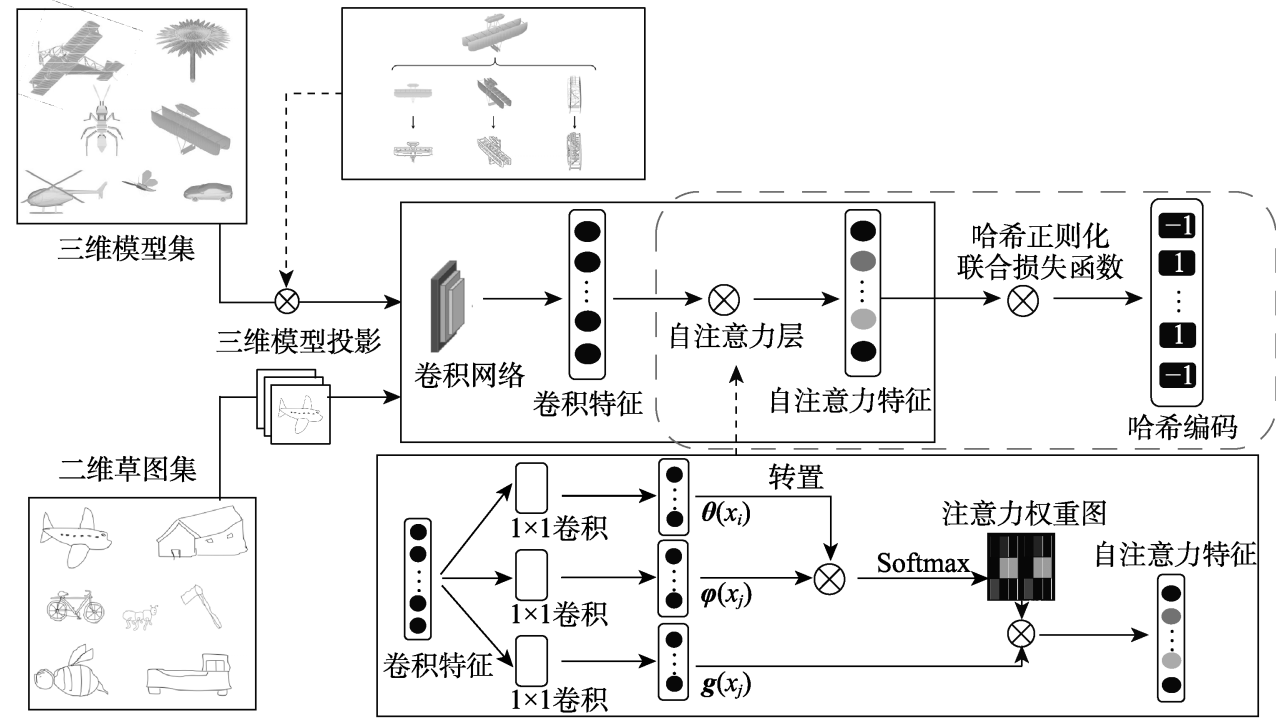

图 1 基于哈希自注意力端到端网络的三维模型草图检索总体框架

处理. 常用的处理方式是采取二维投影即视图的 方式表征三维模型，如全景视图 ${ }^{[13]}$ 和多视图 ${ }^{[14-15]}$ 等. 本文采用文献[16]方法进行投影渲染，得到与 三维模型对应的多个二维视图. 为了简化视点定 义，算法首先采用主成分分析对三维模型进行姿 态归一化处理, 然后将每个模型以 $Z$ 轴为中心轴, 每隔 $\theta$ 角度投影渲染得到不同视点的投影.

为了减少无用特征对检索结果的影响，本文 将上述模型渲染得到的二维视图集采用边缘检测 提取边缘线条图, 如图 2 所示. 此时草图与三维模 型之间的检索问题可以转化为草图与视图之间的 特征匹配问题，在建立草图与视图的共同特征分 布基础上计算草图和三维模型的相似性。

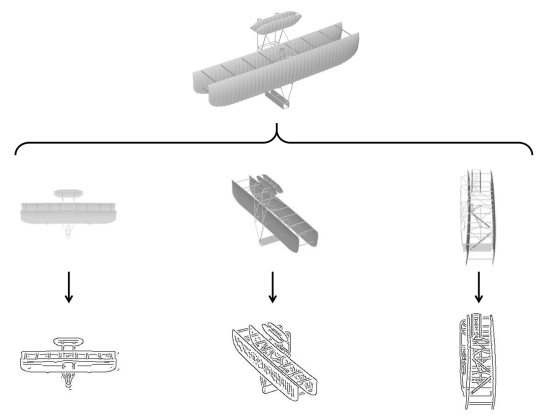

图 2 三维模型投影

\section{2 自注意力层}

算法通过边缘检测得到的视图，尽管在视觉 上呈现和草图类似的结构，但是在局部细节上和 草图上具有很大的特征差异性. 为此, 本文在网络 结构上引人自注意力层 ${ }^{[17-18]}$. 自注意力层对图像 像素的相关性进行卷积学习, 每个像素点位置通
过特征权重提取出物体的重要结构特征. 自注意 力层的公式定义为

$$
y_{i}=\frac{1}{C(x)} \sum_{\forall j} f\left(x_{i}, x_{j}\right) \boldsymbol{g}\left(x_{j}\right) .
$$

其中, $y_{i}$ 为第 $i$ 处特征值的特征权重和; $f\left(x_{i}, x_{j}\right)$ 为 2 个特征值之间的关联性度量函数，在本文中表 示 2 个特征值的点乘相似度，定义为

$$
f\left(x_{i}, x_{j}\right)=\boldsymbol{\theta}\left(x_{i}\right)^{\mathrm{T}} \boldsymbol{\varphi}\left(x_{j}\right) ;
$$

$\boldsymbol{g}\left(x_{j}\right)$ 表示对 $x_{j}$ 点的特征线性映射, 定义为

$$
\boldsymbol{g}\left(x_{j}\right)=\boldsymbol{W}_{g} x_{j} .
$$

其中, $W_{g}$ 为权重学习矩阵; $C(x)$ 为归一化操作 函数, 定义为 $C(x)=N$. 其中, $N$ 为特征值的维 度大小.

考虑自注意力层的最终目的是提取图像的结 构特征值, 而大步长卷积核不仅增加了参数量, 还 易导致在特征映射时丢失结构特征. 因此，本文采 用 $1 \times 1$ 卷积操作分别对 $\boldsymbol{g}\left(x_{j}\right), \boldsymbol{\theta}\left(x_{i}\right)$ 和 $\boldsymbol{\varphi}\left(x_{j}\right)$ 进 行恒等线性映射, 得到卷积特征. 然后将 $\boldsymbol{\theta}\left(x_{i}\right)$ 转 置后与 $\varphi\left(x_{j}\right)$ 矩阵乘积得到注意力权重图, 再将 $\boldsymbol{g}\left(x_{j}\right)$ 的特征向量与经过 Softmax 操作的注意力权 重图进行矩阵乘积, 最终得到经过自注意力加强 距离依赖的特征图. 图 3 所示为卷积网络采用自注 意力层前后所得到的特征可视化效果. 本节以飞 机草图和飞机视图的前 19 维特征进行比较分析. 可以看出, 飞机草图和飞机视图在全局特征结构 信息上具有明显的相似性，但是在局部特征上差 
异性明显. 通过自注意力层的卷积训练, 两者的特 征聚类性得到了加强(如图 3 中橙色线所示), 局部 特征更为接近.

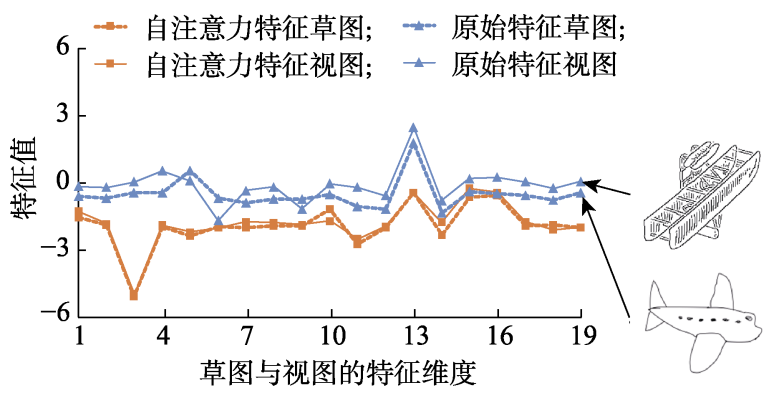

图 3 自注意力特征与原始特征的比较

\section{3 哈希正则化联合损失函数}

在上述自注意力层作用下，网络得到草图和 边缘视图的自注意力特征向量. 考虑采用联合损 失函数定义目标误差, 实现嵌人特征的逼近. 常用 的方法有对比度量学习、三元组度量学习等 ${ }^{[19]}$, 对 比度量学习相比三元组度量学习能够更加严格地 聚类同类样本, 可以得到更为精确的结果. 因此, 本文采用对比度量学习进行特征嵌人，同时考虑 哈希正则化和交叉熵对网络的影响, 形成的联合 损失函数定义为 $L=L_{\mathrm{co}}+\gamma L_{\mathrm{ha}}+\eta L_{\mathrm{ce}}$. 其中， $L_{\mathrm{co}}$ 为 对比损失函数; $L_{\mathrm{ha}}$ 为哈希正则化损失函数; $L_{\mathrm{ce}}$ 为交叉熵损失函数; $\gamma$ 和 $\eta$ 分别为哈希正则化损失 函数和交叉熵损失函数的加权参数.

\subsection{1 对比损失函数}

对比损失函数最初由 Hadsell 等 ${ }^{[20]}$ 提出, 该函 数的输人通常为一对图像对, 其基本准则为同类 样本之间的距离越小越好, 不同类样本之间的距 离如果小于阈值 $m$, 则通过互斥使其距离接近 $m$, 定义为

$$
L_{\mathrm{co}}\left(f_{1}, f_{2}, y\right)=\frac{1}{2}(1-y) D_{\mathrm{w}}{ }^{2}+\frac{1}{2} y\left\{\max \left(0, m-D_{\mathrm{w}}\right)\right\}^{2} .
$$

其中, $f_{1}$ 和 $f_{2}$ 分别为样本对的高层语义特征; $y$ 为样本对的监督信息, 样本对为同类时 $y=0$, 为 不同类时 $y=1 ; D_{\mathrm{w}}$ 为样本对之间的欧几里得度 量, 其定义为 $D_{\mathrm{w}}=\sqrt{\sum_{i=1}^{n} \sum_{j=1}^{n}\left(f_{i}-f_{j}\right)^{2}}$.

当 $y=0$ 时, 调整参数最小化 $f_{1}$ 与 $f_{2}$ 之间的距 离. 当 $y=1$ 时, 如果 $f_{1}$ 与 $f_{2}$ 之间的距离大于 $m$, 则不做优化; 如果 $f_{1}$ 与 $f_{2}$ 之间的距离小于 $m$, 则 增大两者距离到 $m$.

\subsection{2 哈希正则化损失函数}

在草图检索三维模型的过程中, 直接对原始 特征进行计算需要耗费大量计算代价. 为了有效 地降低草图检索成本, 研究人员常常将卷积网络 所提取的高层语义特征映射为紧凑的二进制编码， 但仍保留图像原始的语义信息 ${ }^{[11,21]}$. 常用的哈希 方法是直接使用非线性函数(如 Sigmoid 等), 将输 出层的特征转为二进制编码, 但是这些函数很容 易丢失图像原本的有益信息. 针对这个问题，哈希 正则化损失能够将实值输出约束成类二进制编码, 在输出图像的原始特征同时保持特征的二值性. 为此, 本文提出在对比度量学习的基础上嵌人哈 希正则化约束, 构造一个新的加权度量学习函数. 本文在哈希损失函数中采用 $L_{1}$ 正则化对特征输出 进行二进制的约束, 使特征输出约束为接近 -1 或 1 的类二进制编码, 公式定义为 $L_{\mathrm{ha}}=\left\|\left|f_{i}\right|-1\right\|_{1}$. 其 中, $f_{i}$ 为卷积网络输出的高层语义特征. 该函数 可以在学习共同特征分布的同时, 将最终的特征 输出约束为类二进制码, 而后在检索过程中使用 激活函数(如 $\operatorname{sgn}$ 函数), 将得到的语义特征映射为 哈希编码。

\subsection{3 交叉熵损失函数}

为了得到更加紧凑的图像特征, 本文进一步 在上述加权损失函数的基础上加人交叉熵损失函 数, 该函数能够衡量深度卷积网络的预测值与实 际值的差异性信息, 即能够有效地约束同类样本 的特征分布距离. 其定义为 $L_{\mathrm{ce}}=-\sum_{j=1}^{n} y_{j} \ln \left(f_{j}\right)$. 其 中, $n$ 为类别数量; $y_{j}$ 为样本 $j$ 的类别标签; $f_{j}$ 为样本 $j$ 由网络输出的分类特征.

综上, 基于对比度量学习的加权损失函数在 嵌人交叉熵损失函数和哈希正则化约束后, 能够 有效形成同类数据间不同域数据以及相同域数据 的共同特征分布，同时提高了特征嵌人学习的速 度以及准确率。

\section{2 实验结果与分析}

本节对算法进行实验分析, 采用的基准数据 集为 SHREC13 ${ }^{[22]}$ 和 SHREC14 ${ }^{[23]}$, 它们是目前用 于三维模型草图检索的标准测试集. 首先对每个 三维模型进行投影渲染, 将渲染所得的二维视图 标记为与三维模型同样的类别标签, 并将二维视 
图集全部投人到后续训练过程中. 本文和已有不 同类型的检索算法进行了比较，采用的评价性能 指标分别为：最近邻(nearest neighbor, NN)、第 1 批次(first tier, FT)、第 2 批次 (second tier, ST)、E 方法 (e-measure，E)、折扣累计收益 (discounted cumulative gain, DCG)及平均准确率 (mean average precision, MAP $)^{[22]}$. 本节首先给出网络结构细节, 然后对算法进行消融性实验，以验证各模块对检 索性能的影响，最后给出不同算法的检索性能.

\section{1 网络结构细节}

本文采用深度卷积网络提取草图和视图的高 层语义特征. 网络预训练数据集为 ImageNet, 特 征提取模块采用 seResNeXt-101 网络 ${ }^{[24]}$. 整个网络 结构采用哈希权重共享网络同时训练草图和视图, 将网络的深度卷积特征截取在全连接层之前. 然 后将输出的深度卷积特征输人自注意力层, 得到 自注意力特征; 将自注意力特征输人池化层, 并通 过 3 层全连接层降维, 得到最终的语义特征. 本文 将最终的输出维度设置为 256 维. 整体网络结构如 表 1 所示, 其中, $K$ 为卷积核尺寸, $S$ 为步长, $D$ 为输出维度, $F$ 为激活函数.

表 1 网络结构

\begin{tabular}{|c|c|c|}
\hline 层类别 & 参数 & 输出尺寸 \\
\hline \multirow{5}{*}{ 卷积层 } & $K=7, S=2$ & 64 \\
\hline & seResNeXtBlock $\times 3$ & 128 \\
\hline & seResNeXtBlock $\times 4$ & 256 \\
\hline & seResNeXtBlock $\times 23$ & 1024 \\
\hline & seResNeXtBlock $\times 3$ & 2048 \\
\hline 自注意力层 & $D=2048, F=$ 'ReLU' & 2048 \\
\hline 池化层 & $K=1$ & 2048 \\
\hline \multirow{3}{*}{ 全连接层 } & $D=1024, F=$ 'ReLU' & 1024 \\
\hline & $D=512, F=$ 'ReLU' & 512 \\
\hline & $D=256$ & 256 \\
\hline
\end{tabular}

实验中一个批次大小为 20 , 共迭代 100 次进 行训练, 最终输出的特征维度设置为 256 维, 加权 损失函数的参数设置为 $1,0.01,10$, 采用自适应矩 估计优化器(简称 Adam 优化器)进行优化, 指数衰 减率设置为 $(0.9,0.9)$, 初始学习速率为 0.0001 , 采 用余弦学习率衰减方法.

\section{2 消融性实验}

\subsection{1 卷积网络特征维度的选择}

不同维度的特征所能囊括的图像信息不同, 过小维度的特征无法涵盖所有的信息，过大维度 的特征又会造成信息的过度复用而难以收敛。因
此, 本文对不同维度的特征进行了对比实验, 实验 结果如表 2 所示. 可以看出, 最优维度的特征为 256 维, 更大维度的特征没有得到更优的结果, 反 而增加了内存占用和计算时间.

表 2 特征维度对实验结果的影响

\begin{tabular}{cc}
\hline 特征维度 & MAP \\
\hline 128 & 0.859 \\
256 & 0.875 \\
512 & 0.806 \\
\hline
\end{tabular}

\subsection{2 类间距离的阈值参数的选择}

本文所设计的加权损失函数除了各损失函数 的加权参数以外，还通过阈值 $m$ 控制类间距离的 大小. 若 $m$ 选择过小, 样本会因类内距离大于类 间距离而导致特征不可分; $m$ 选择过大又会牺牲 空间导致无法聚类. 实验结果如表 3 所示, 可以看 出, 当 $m=2$ 时, 所得到的实验结果最优.

\section{表 3 距离阈值对实验结果的影响}

\begin{tabular}{ll}
\hline$m$ & MAP \\
\hline 1 & 0.860 \\
2 & 0.875 \\
3 & 0.840 \\
6 & 0.807 \\
\hline
\end{tabular}

\subsection{3 不同模块对实验结果的影响}

相比直接提取原始特征进行特征嵌人和相似 性评价, 将特征转化为哈希编码有着减少内存占 用和提高收玫速度的优势. 另外, 语义标签约束下 的哈希正则化学习通过类二进制编码保持草图和 边缘视图的共性形状特征, 而忽略了局部特征. 因 此, 通过自注意力特征该损失项可明显提高两者 之间的类内聚类性. 实验结果如表 4 所示, 可以看 出, 加人哈希正则化和自注意力层能够提高检索 准确率.

表 4 各模块对实验结果的影响

\begin{tabular}{ll}
\hline \multicolumn{1}{c}{ 对比实验 } & MAP \\
\hline with 哈希/自注意力层 & 0.875 \\
with 哈希 & 0.867 \\
with 自注意力层 & 0.846 \\
without 哈希/自注意力层 & 0.838 \\
\hline
\end{tabular}

\subsection{4 视点采样角度 $\theta$ 对实验结果的影响}

为了观察不同视图个数对网络效果的影响, 本文通过控制视点采样角度 $\theta$ 得到不同的投影视 
图个数进行实验分析. 不同视图个数下所得到的 实验结果如图 4 所示. 可以看出, 当视图个数从 1 变化到 3 时检索性能有明显的提高，主要是由于用 户在绘制草图时具有一定的主观性，用户绘制的 草图和视图具有多对一问题. 因此, 采用单一视图 会导致部分草图无法得到很好的检索效果．但是 随着视图个数的增加, 检索性能也并不是线性上 升, 主要原因是视图个数增加到一定量时, 其不同 方向的视图已经能够反映用户绘制的草图，从而 导致检索性能趋于稳定

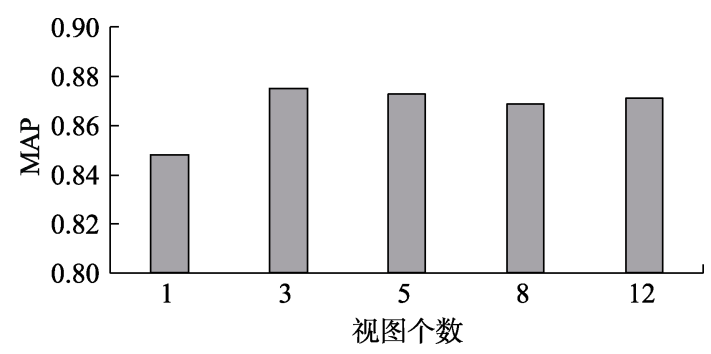

图 4 视图个数对结果的影响

\section{3 实验比较}

\subsubsection{SHREC13 数据集实验结果比较}

为了进一步验证算法的有效性，本文与已有 的三维模型草图检索算法 Siamese $^{[8]}, \mathrm{LWBR}^{[9]}$, $\mathrm{DCHML}^{[6]}, \mathrm{DCA}^{[10]}$ 和 $\mathrm{JFM}^{[12]}$ 进行性能比较. 为了 比较的一致性, 本文没有对训练数据进行扩增, 只 是通过基准集提供的训练数据完成训练. 不同算 法所得到的结果如表 5 所示.

表 5 SHREC13 数据集性能对比

\begin{tabular}{lcccccc}
\hline \multicolumn{1}{c}{ 算法 } & NN & FT & ST & E & DCG & MAP \\
\hline Siamese $^{[8]}$ & 0.405 & 0.403 & 0.548 & 0.287 & 0.607 & 0.469 \\
LWBR $^{[9]}$ & 0.712 & 0.725 & 0.785 & 0.369 & 0.814 & 0.752 \\
DCHML $^{[6]}$ & 0.730 & 0.715 & 0.773 & 0.368 & 0.816 & 0.744 \\
DCA $^{[10]}$ & 0.783 & 0.796 & 0.829 & 0.376 & 0.856 & 0.813 \\
JFM $^{[12]}$ & 0.848 & 0.775 & 0.862 & 0.409 & 0.890 & 0.818 \\
本文 & 0.839 & 0.858 & 0.903 & 0.420 & 0.904 & 0.875 \\
\hline
\end{tabular}

由表 5 可得, 本文算法在 SHREC13 数据集上 的部分评价指标上优于以往的检索算法, 包括 2019 年白静等 ${ }^{[12]}$ 提出的联合特征映射算法(JFM). 主要原因是 JFM 算法在特征描述上采用传统的 Alex 网络结构, 这种网络结构只进行局部卷积操 作，而没有如自注意力层一般进行全局相关性卷 积操作. 因此, 本文算法提取得到的特征更好地反 映出草图和边缘视图的共性结构特征, 且能忽略 手绘草图在局部线条上的不准确. 另外，本文设计
的哈希正则化联合损失函数能够有效完成同类数 据间不同域数据与相同域数据的共同特征映射, 提高了草图和视图的相似性和可分性.

图 5 所示为部分检索算法在 SHREC13 数据集 上的准确率-召回率(precision-recall, PR)曲线. 可 以看出，本文算法在所有召回率 $R$ 条件下的准确 率 $P$ 均超过 $80 \%$; 本文算法在不同召回率 $R$ 上的 准确率 $P$ 均高于其他算法.

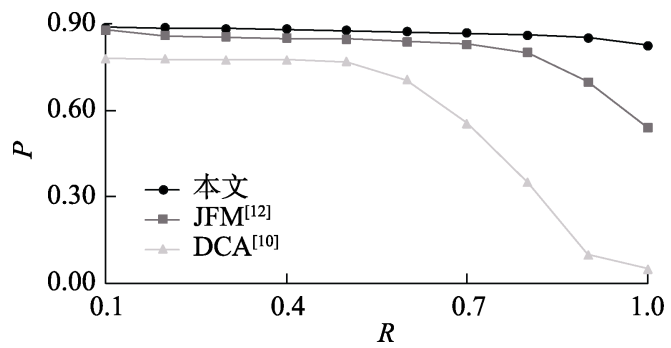

图 5 在 SHREC13 数据集上的 PR 曲线对比

\subsubsection{SHREC14 数据集实验结果比较}

为了进一步验证算法的检索性能, 本文也给出 了在 SHREC14 数据集上的实验结果, 如表 6 所示.

表 6 SHREC14 数据集性能对比

\begin{tabular}{lcccccc}
\hline \multicolumn{1}{c}{ 算法 } & NN & FT & ST & E & DCG & MAP \\
\hline Siamese $^{[8]}$ & 0.239 & 0.212 & 0.316 & 0.140 & 0.496 & 0.228 \\
LWBR $^{[9]}$ & 0.403 & 0.378 & 0.455 & 0.236 & 0.581 & 0.401 \\
DCHML $^{[6]}$ & 0.403 & 0.329 & 0.394 & 0.202 & 0.544 & 0.336 \\
$\mathrm{JFM}^{[12]}$ & 0.835 & 0.720 & 0.810 & 0.390 & 0.873 & 0.756 \\
$\mathrm{DCA}^{[10]}$ & 0.770 & 0.789 & 0.823 & 0.398 & 0.859 & 0.803 \\
本文 & 0.777 & 0.794 & 0.836 & 0.405 & 0.869 & 0.812 \\
\hline
\end{tabular}

由表 6 可得, 本文算法在 SHREC14 数据集上 的部分评价指标上优于以往的检索算法. 类似地, 和 JFM 算法相比较, 本文算法在 MAP 指标上也得 到了明显的提高.

图 6 所示为部分检索算法在 SHREC14 数据集 上的 PR 曲线，可以看出，本文算法在 $70 \%$ 100\% 的召回率 $R$ 范围的准确率 $P$ 均高于其他算法, 且 整体准确率 $P$ 超过 $78 \%$ ，具有稳定性。

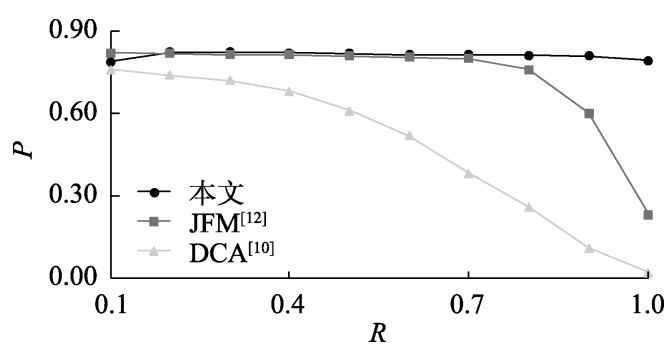

图 6 在 SHREC14 数据集上的 PR 曲线对比 


\section{4 可视化检索效果}

表 7 所示为部分基于草图的三维模型检索结 果，对于测试集中的 6 张不同类的手绘草图，本文 算法按照相似度从大到小排序，在草图对应行的 右侧给出了检索到的三维模型; 最后一行所示的 部分用黑色边框框出的是算法检索错误的例子. 可以看出, 这种错误的检索结果主要原因是手绘 草图的歧义性, 即使是人也难以区分长登与桌子, 因此也就难以由手绘草图检索到准确的三维模型. 对于这方面工作，很难采用几何特征进行区分. 在 后续工作中, 如何在草图检索融人语义信息, 形成 多特征融合下的三维模型草图检索框架，是本文 需要进一步考虑的问题.

\section{表 7 基于草图的三维模型检索结果展示}

手绘草图

\section{3 结 语}

本文提出一种基于哈希自注意力的端到端三 维模型草图检索算法. 算法利用哈希正则化联合 损失函数得到特征编码，同时在网络中嵌人自注 意力层，提高特征聚类性. 实验表明，本文算法所 建立的三维模型草图检索网络，在有效聚类同类 数据的特征的同时减少数据域间差异性，能够实 现较为准确的检索目标.

在后续工作中，可以考虑在已有网络框架中 引人细粒度模型，通过视图或草图的局部特征进 一步提高网络的准确率. 另外，由于草图的绘制过 程具有主观性，而用户的文本描述又无法准确地 表达检索需求. 在后续研究中，可以考虑采用文本 信息和草图建立联合检索框架，进一步提高三维 模型草图检索的准确性和可靠性

\section{参考文献(References):}

[1] Cui Chenyang, Shi Jiaoying. Analysis of feature extraction in 3D model retrieval[J]. Journal of Computer-Aided Design \& Computer Graphics, 2004, 16(7): 882-889(in Chinese) (崔晨昒, 石教英. 三维模型检索中的特征提取技术综述 [J]. 计算机辅助设计与图形学学报, 2004, 16(7): 882-889)

[2] Li B, Schreck T, Godil A, et al. Shrec'12 track: sketch-based 3D shape retrieval[C] //Proceedings of the 5th Eurographics Conference on 3D Object Retrieval. Aire-la-Ville: Eurographics Association Press, 2012: 109-118

[3] Yu Deng, Liu Yujie, Xing Minmin, et al. Sketch-based image retrieval using cross-domain modeling and deep fusion network[J]. Journal of Software, 2019, 30(11): 3567-3577(in Chinese)

(于邓，刘玉杰，邢敏敏，等. 包含跨域建模和深度融合网络 的手绘草图检索[J]. 软件学报, 2019, 30(11): 3567-3577)

[4] Liu Yujie, Dou Changhong, Zhao Qilu, et al. Sketch based image retrieval with conditional generative adversarial network[J]. Journal of Computer-Aided Design \& Computer Graphics, 2017, 29(12): 2336-2342(in Chinese)

(刘玉杰, 荬长红, 赵其鲁, 等. 基于条件生成对抗网络的手 绘图像检索 [J]. 计算机辅助设计与图形学学报, 2017, 29(12): 2336-2342)

[5] Eitz M, Richter R, Boubekeur T, et al. Sketch-based shape retrieval[J]. ACM Transactions on Graphics, 2012, 31(4): Article No.31

[6] Dai G X, Xie J, Fang Y. Deep correlated holistic metric learning for sketch-based 3D shape retrieval[J]. IEEE Transactions on Image Processing, 2018, 27(7): 3374-3386

[7] Mu P P, Zhang S Y, Zhang Y, et al. Image-based 3D model retrieval using manifold learning[J]. Frontiers of Information Technology \& Electronic Engineering, 2018, 19(11): 1397-1408

[8] Wang F, Kang L, Li Y. Sketch-based 3D shape retrieval using convolutional neural networks[C] //Proceedings of the IEEE Conference on Computer Vision and Pattern Recognition. Los Alamitos: IEEE Computer Society Press, 2015: 1875-1883

[9] Xie J, Dai G X, Zhu F, et al. Learning barycentric representations of 3D shapes for sketch-based 3D shape retrieval[C] // Proceedings of the IEEE Conference on Computer Vision and Pattern Recognition. Los Alamitos: IEEE Computer Society Press, 2017: 3615-3623

[10] Chen J X, Fang Y. Deep cross-modality adaptation via semantics preserving adversarial learning for sketch-based 3D shape retrieval[C] //Proceedings of the European Conference on Computer Vision. Heidelberg: Springer, 2018: 624-640

[11] Chen J X, Qin J, Liu L, et al. Deep sketch-shape hashing with segmented 3D stochastic viewing[C] //Proceedings of the IEEE Conference on Computer Vision and Pattern Recognition. Los Alamitos: IEEE Computer Society Press, 2019: 791-800

[12] Bai Jing, Kong Dexin, Zhou Wenhui, et al. Joint feature mapping for end-to-end sketch-based 3D model retrieval[J]. Journal of Computer-Aided Design \& Computer Graphics, 2019, 31(12): 2056-2065(in Chinese) (白静，孔德馨，周文惠，等. 基于联合特征映射的端到端三 
维模型草图检索 $[J]$. 计算机辅助设计与图形学学报, 2019, 31(12): 2056-2065)

[13] Shi B G, Bai S, Zhou Z C, et al. DeepPano: deep panoramic representation for 3D shape recognition[J]. IEEE Signal Processing Letters, 2015, 22(12): 2339-2343

[14] Ma Y X, Zheng B, Guo Y L, et al. Boosting multi-view convolutional neural networks for $3 \mathrm{D}$ object recognition via view saliency[C] //Proceedings of the Conference on Advances in Image and Graphics Technologies. Heidelberg: Springer, 2017: 199-209

[15] Sinha A, Bai J, Ramani K. Deep learning 3D shape surfaces using geometry images[C] //Proceedings of European Conference on Computer Vision. Heidelberg: Springer, 2016: 223-240

[16] Su H, Maji S, Kalogerakis E, et al. Multi-view convolutional neural networks for 3D shape recognition[C] //Proceedings of the IEEE International Conference on Computer Vision. Los Alamitos: IEEE Computer Society Press, 2015: 945-953

[17] Zhang H, Goodfellow I, Metaxas D, et al. Self-attention generative adversarial networks[C] //Proceedings of the 36th International Conference on Machine Learning. New York: ACM Press, 2019: 7354-7363

[18] Wang X L, Girshick R, Gupta A, et al. Non-local neural networks[C] //Proceedings of the IEEE Conference on Computer Vision and Pattern Recognition. Los Alamitos: IEEE Computer
Society Press, 2018: 7794-7803

[19] Li Q, Sun Z N, He R, et al. Deep supervised discrete hashing[C] //Proceedings of the 31st International Conference on Neural Information Processing Systems. New York: ACM Press, 2017: 2479-2488

[20] Hadsell R, Chopra S, LeCun Y. Dimensionality reduction by learning an invariant mapping[C] //Proceedings of the IEEE Conference on Computer Vision and Pattern Recognition. Los Alamitos: IEEE Computer Society Press, 2006: 1735-1742

[21] Liu H M, Wang R P, Shan S G, et al. Deep supervised hashing for fast image retrieval[C] //Proceedings of the IEEE Conference on Computer Vision and Pattern Recognition. Los Alamitos: IEEE Computer Society Press, 2016: 2064-2072

[22] Li B, Lu Y J, Godil A, et al. SHREC'13 track: large scale sketch-based 3D shape retrieval[C] //Proceedings of Euro Graphics Workshop on 3D Object Retrieval. Aire-la-Ville: Euro Graphics Association Press, 2013: 89-96

[23] Li B, Lu Y J, Godil A, et al. A comparison of methods for sketch-based 3D shape retrieval[J]. Computer Vision and Image Understanding, 2014, 119: 57-80

[24] Xie S N, Girshick R, Dollár P, et al. Aggregated residual transformations for deep neural networks[C] //Proceedings of the IEEE Conference on Computer Vision and Pattern Recognition. Los Alamitos: IEEE Computer Society Press, 2017: 1492-1500 